\title{
Cardioprotective effects of fetal kidney-derived mesenchymal stem cells on doxorubicin-induced cardiotoxicity in rats
}

\author{
ORHAN YAVUZ¹, BAŞAK BOZTOK ÖZGERMEN², ALI EVREN HAYDARDEDEOĞLU³ \\ GÜNGÖR ÇAĞDAŞ DINÇEL ${ }^{4}$
}

\author{
${ }^{1}$ Department of Pathology, ${ }^{2}$ Department of Surgery, ${ }^{3}$ Department of Internal Medicine, \\ Faculty of Veterinary Medicine, Aksaray University, 68100, Aksaray, Turkey \\ ${ }^{4}$ Veterinary Department, Eskil Vocational School, Aksaray University, 68100, Aksaray, Turkey
}

Yavuz O., Boztok ÖzgermenB., Haydardedeoğlu A. E., Çağdaş Dinçel G. Cardioprotective effects of fetal kidney-derived mesenchymal stem cells on doxorubicin-induced cardiotoxicity in rats

\section{Summary}

Cardiotoxicity is one of the most common side effects of doxorubicin (DOX), a chemotherapy drug used in the treatment of many carcinomas. In recent years, stem-cell therapies have been successfully used to prevent cardiotoxicity. This study investigated the efficacy of intraperitoneally administered fetal kidney-derived mesenchymal stem cells (FKD-MSCs) in preventing DOX-induced cardiotoxicity in rats. For this purpose, thirty rats were randomly divided into three groups: control, DOX and mesenchymal stem cell (MSC) groups. Adriamycin was injected as a single dose via the tail vein in the DOX and MSC groups in order to induce cardiotoxicity. FKD-MSC was applied to the MSC group by the intraperitoneal route after cardiotoxicity had been established. Then the rats were euthanized, and routine histological procedures were performed on their hearts. H\&E and Masson's stains were used for histopathology. Cardiac Troponin-T and I (cTnT, cTnI), Caspase-3 and BCL-XL antibodies were used for immunohistochemistry. Vacuoles, edema, degeneration and necrosis were observed histopathologically mostly in the DOX group. Lesions in the control and MSC groups were less severe. Fibrosis in the control and MSC groups was milder. cTnT and cTnI immunopositive staining was most commonly seen in the control group, followed by the MSC group. Immunohistochemical staining by Caspase-3 and BCL-XL showed that their expressions in the MSC group were statistically similar to those in the control group. Accordingly, it was concluded that the intraperitoneal application of MSC had a positive effect on histopathological findings, fibrosis, immunohistochemistry, especially apoptosis, neovascularization, and anti-apoptotic development, whereas troponin levels were not found to be therapeutic.

Keywords: apoptosis, cardiotoxicity, doxorubicin, stem cell, troponin

Doxorubicin (DOX) is an antineoplastic agent of the anthracyclin group. It is widely used against solid and haematological cancer types, such as lymphoma, thyroid tumours and hemangiosarcoma. However, it has been reported that there are many side effects, especially cardiotoxicity (3). DOX-induced cardiotoxicity (Dox-IC) occurs as acute, subacute or chronic cardiomyopathy (23). It has been reported that both acute and chronic toxicities may cause cardiac dysfunction, heart failure, and even death (19). Although the molecular mechanisms of DOX in cardiotoxicity have not been fully elucidated, reactive oxygen species, cardiac oxidative stress, disruption of myofibrils, and intracellular calcium dysregulation result in cell death by necrosis and apoptosis in cardiomyocytes (13).
DOX causes autophagy, necrosis and apoptosis by increasing mitochondrial damage and iron-rich lysosome rupture in the Reactive Oxygen Species (ROS)-iron phenomenon, which is the main mechanism of Dox-IC. It has also been reported that DOX triggers apoptosis by increasing the expression of the p53 gene in cells and causing cell senescence. In the mechanism of the ubiquitin-proteasome system (UPS), it causes apoptosis in the cells by upregulation of proapoptotic factors. Activation of the UPS also leads to the reduction of myofilament proteins, such as $\beta$-catenin, resulting in cardiomyopathy (16).

Mesenchymal stem cells (MSC), known as adult stem cells, are capable of regenerating and differentiating themselves, as other stem cells (20). Stem cell 
therapy can be used to prevent many diseases, and it has been reported that DOX-related cardiomyopathies have been the subject of experimental studies in recent years. MSCs activate some mechanisms, such as anti-fibrotic, anti-apoptotic and extracellular matrix remodeling, by releasing paracrine factors, immunomodulators and metallopeptides in cardiac tissue. These MSCs also inhibit oxidative stress and prevent apoptosis by increasing their antioxidant enzyme and anti-apoptotic activity (1). These features make MSCs useful in treating cardiac damage (15). Although there are many reports in the literature on the effects of different administration routes of stem cells, most are restricted to intracardiac, intravenous and intramuscular administration routes. It has been noted that MSCs that enter the systemic circulation intravenously can easily reach the cardiac tissue, but the intravenous route was insufficient if a certain period of time had passed after cardiac damage (15). Intracoronary and epimyocardial routes, especially in experimental studies, are relatively difficult to use on rats, and alternative administration methods have been sought. It has been observed that the intraperitoneal administration route is easier to use than the above-mentioned, and it can be an alternative to other methods $(1,7)$.

In a previous study, it was reported that MSCs originating from fetal kidneys differentiate into adipose tissue, hepatocytes and chondrocytes, and they have potential benefits in medical treatment (8). However, there are not many studies on the use of fetal kidneyderived mesenchymal stem cells (FKD-MSCs) to prevent cardiac damage. The aim of this study was to investigate the effects of FKD-MSCs on histopathological findings, such as vacuoles, hyaline degeneration and Zenker's necrosis, as well as the expressions of apoptosis and anti-apoptotic factors, such as Caspase-3 and BCL-XL, in experimental Dox-IC in the myocardial tissues of rats.

\section{Material and methods}

Obtaining fetal kidney-derived mesenchymal stem cells. The method of Pinarli et al. (14) was used to obtain the stem cells and to design the experimental groups. In order to obtain FKD-MSCs, hysterectomy was performed on 19-day-old pregnant rats by median line laparotomy under sterile conditions, and fetuses were taken. The fetuses were anesthetized and euthanized by ether, and kidney tissues were removed and stored in Dulbecco's Modified Eagle's Medium (DMEM).

The fetal kidney tissues were mechanically dissected into small pieces with a sterile scalpel. Then, tissues obtained by the explant culture method were placed in T25 flasks and incubated at $37^{\circ} \mathrm{C}$ in $5 \% \mathrm{CO}_{2}$ humidified atmosphere. $77 \%$ DMEM, 20\% fetal bovine serum-FBS, 2\% L-glutamine, 1\% penicillin, streptomycin, and amphotericin were added to the medium. The medium was changed once every 2-3 days to remove non-adherent cells. When about $70 \%$ adhesion was present, adherent MSCs were passaged and aliquoted
$1: 2$ with $0.25 \%$ Trypsin in FBS. The cells were grown to passage 3 . To evaluate the differentiation potential of FKDMSCs, the cells were seeded at a density of $5 \times 10^{4}$ cells $/ \mathrm{cm}^{2}$ in a six-well culture plate at $\mathrm{P} 3$ and differentiated into adipogenic, chondrogenic or osteogenic differentiation media for $5 \% \mathrm{CO}_{2}$ and $37^{\circ} \mathrm{C}$ for 21 days. Then, oil red, Von Kossa and alcian blue stainings were performed to demonstrate differentiation into fat cells, osteoblasts and chondroblasts, respectively. Phenotypic characterization of FKD-MSCs was done by flow cytometry. For this purpose, the cells were incubated in antibodies in PBS containing 1\% fetal bovine serum for $30 \mathrm{~min}$ at $4^{\circ} \mathrm{C}$. Then, flow cytometry was performed by Beckman Coulter CytoFLEX and analysed. Analysis of flow cytometry revealed that the cells isolated from fetal kidney tissue expressed CD90 and CD105, but not CD34 and CD45. The FKD-MSCs obtained were used for in vivo transplantation.

Experimental groups. Thirty male Sprague-Dawley rats weighing $200 \pm 20 \mathrm{~g}$ were used in this study. All rats were kept under standard laboratory conditions $\left(21 \pm 2^{\circ} \mathrm{C}\right.$, $65 \%$ humidity and $12 \mathrm{~h}$ light $/ 12 \mathrm{~h}$ dark). The animals were fed ad libitum and allowed access to water continuously. Randomly selected 10 animals were divided into Control, DOX and MSC groups. In order to induce cardiotoxicity, the rats received a single tail vein injection of Adriamycin dissolved in $10 \mathrm{mg} / \mathrm{kg} / \mathrm{LW}+0.9 \% \mathrm{NaCl}$. The experimental groups were given the following treatments:

Control group: Initially, $10 \mathrm{mg} / \mathrm{kg} 0.9 \% \mathrm{NaCl}$ was administered as a single dose into the tail vein. Seven days later, $0.9 \% \mathrm{NaCl}$ in the same volume was administered intraperitoneally 3 times at one week intervals.

DOX group: After 7 days of cardiotoxicity, $0.9 \% \mathrm{NaCl}$ in the same volume as FKD-MSCs was administered intraperitoneally 3 times at one week intervals.

MSC group: After 7 days of cardiotoxicity, $2 \times 10^{6}$ doses of FKD-MSCs were administered by intraperitoneal injection 3 times at one week intervals.

The rats were observed for 5 weeks after the end of treatment and euthanized by decapitation under xylazine/ ketamine general anesthesia.

Histopathological method. The rats were necropsied, and their hearts were removed and kept in a $10 \%$ buffered formalin solution for 24 hours. Sections for histological examination were cut from the middle part of the heart containing ventricular layers. Subsequently, tissues were passed through alcohol, xylol and paraffin series and then embedded in paraffin wax for routine histological tissue processing. The paraffin blocks were cut into $5 \mu \mathrm{m}$ thick slices by microtome and examined under a light microscope after staining with Haematoxylin-Eosin (HE) and Masson's trichrome stain (9).

Histopathological scoring. Histopathological evaluation was performed semi-quantitatively. Previous studies were used in the histological evaluation of cardiac tissue (4). Accordingly, cardiac muscle tissue fibers were evaluated for vacuolization, hyaline degeneration and Zenker's necrosis as follows:

0 , if no changes were observed in the slide,

(Minimal) +1 , if changes were limited to only one region of the slide, 
(Mild) +2 , if pathological changes showed multifocal distribution in the slide, but in limited areas,

(Moderate) +3 , if pathological changes showed multifocal distribution in the slide in wider areas,

(Severe) +4 , if pathological changes showed diffuse distribution.

The fibrosis score observed in Masson's trichrome staining was taken into account as a percentage by the ImageJ (V1.52a) program. For this purpose, 10 images of each section were taken at $400 \times$ magnification, and the mean value of staining percentages was obtained.

Immunohistochemical method. The indirect immunoperoxidase method was used in immunohistochemical examinations. For this purpose, $5 \mu \mathrm{m}$ thick sections of paraffin wax were attached to polylysine slides. The sections were dried for $15 \mathrm{~min}$ in an oven at $60^{\circ} \mathrm{C}$. Subsequently, they were passed through xylol5 3 times for $5 \mathrm{~min}$ and then through $96 \%, 90 \%, 80 \%, 70 \%$ and $50 \%$ alcohols for deparaffinization. Subsequently, for antigen retrieval, the sections were boiled in citrate buffer solution for $20 \mathrm{~min}$. Then, $3 \% \mathrm{H}_{2} \mathrm{O}_{2}$ was added to the sections to remove peroxidase activity and incubated for $20 \mathrm{~min}$. Block solution was dropped on the slides for $5 \mathrm{~min}$, and primary antibodies [Anti-Cardiac Troponin I Antibody (Santa Cruz Biotechnology, inc. sc-133117), Anti-Cardiac Troponin T Antibody (Abcam ab8295), Anti-Caspase-3 Antibody (Santa Cruz Biotechnology, inc. sc-7272), Anti-BCL-LX Antibody (Santa Cruz Biotechnology, inc. sc-8392)] were instilled and incubated for $2 \mathrm{~h}$. At the end of incubation, biotinylated secondary antibody and streptavidin solution were instilled for $15 \mathrm{~min}$. Finally, the sections were stained with aminoethyl carbasole (AEC) and counterstained with hematoxylin. All stained sections were examined under a light microscope.

Immunohistochemical scoring. The ImageJ (V1.52a) program was used for immunohistochemical scoring of the slides. A total of 15 photographs of each section, including the left ventricular wall, right ventricular wall and interventricular septum, were taken at $400 \times$ magnification. Immunohistochemical staining surface areas in muscle filaments were obtained as percentages. The mean value of 10 staining surface areas of each case was accepted as the score of one case. In order to prevent errors during scoring, only muscles were included in the photographs.

Statistical analysis. Statistical analyses were performed by the IBM ${ }^{\circledR}$ SPSS $^{\circledR}$ Statistics Version 22 program. The significance of histopathological findings was determined by ANOVA and Tukey tests, and the significance of immunohistochemical findings was determined by Kruskal Wallis and Tamhane tests. $\mathrm{P}<0.05$ was accepted as an indicator of statistically significant differences between the groups.

Ethical considerations. The study was performed according to the internal regulations of the Ministry of Agriculture and Forestry of the Republic of Turkey. It also complied with the universal ethical standards (Ethical Committee of Dişkapı Yildirim Beyazit Training and Research Hospital, Approval number 2014/55).

\section{Results and discussion}

Histopathological results. Statistical results and histopathological findings are given in Figure 1A-D. Although vacuole formation was observed in 2 cases in the control group and in 6 cases in the MSC group, it was present in all cases in the DOX group and was more severe. Vacuoles were observed mostly in endocardium and muscle fibers located close to the cavities of ventricles. In some cases, large vacuoles could be detected at small magnifications.

Hyaline degeneration in the control group was minimal. In the MSC group, it was mild, and minimal changes were observed. In the DOX group, hyaline degeneration was observed in all cases. Multifocal lesions were also observed in larger areas. Degenerative

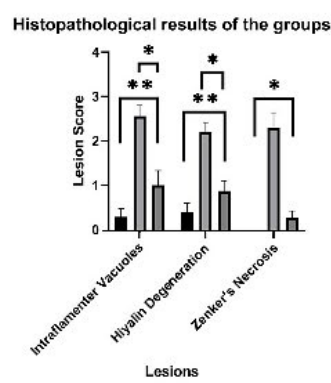

Masson's Trichrome Stain
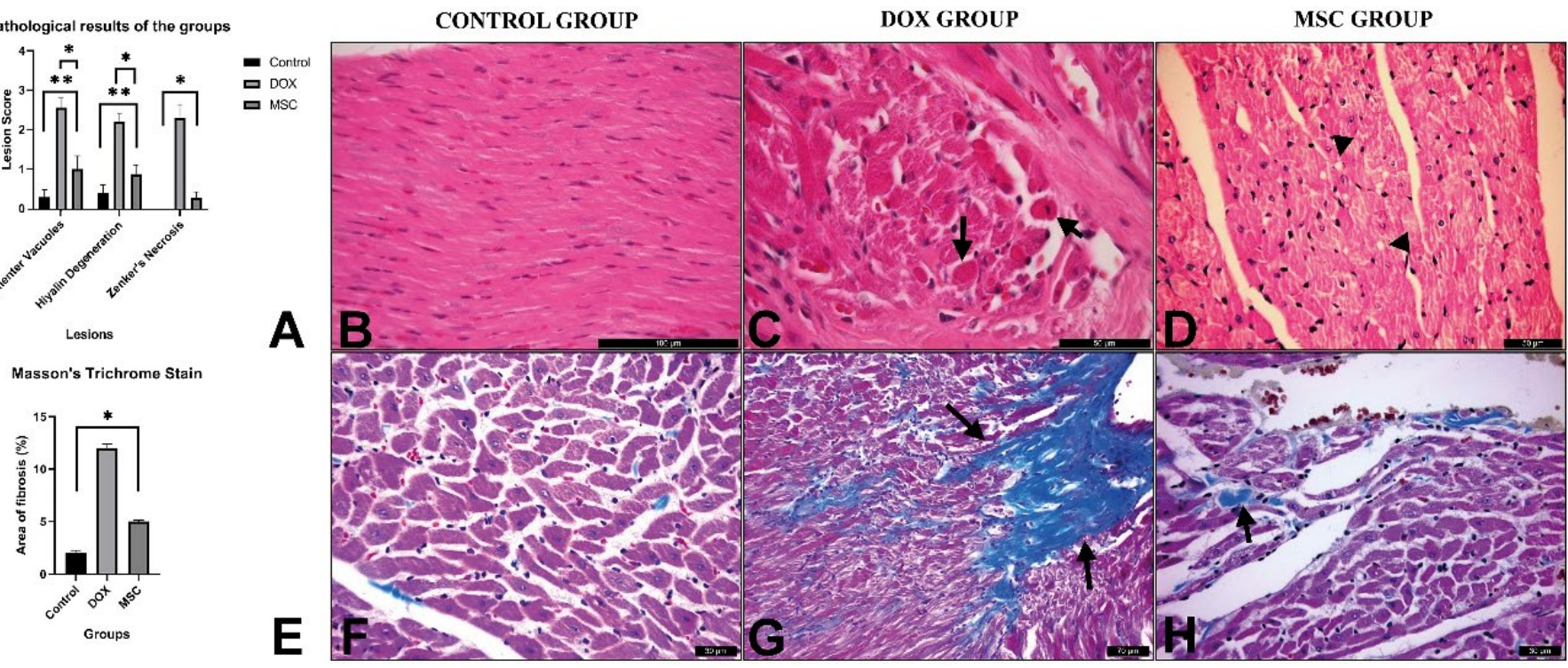

Fig. 1. A) The histopathological results of the groups. B) Control group. HE. C) Zenker's necrosis (arrows) in the muscle filaments in the DOX group. HE. D) Vacuole formations in muscle cells (arrowheads), HE. E) Massons's trichrome stain results of the groups. F) Control group. Masson's stain. G) Wide fibrosis area in muscle filaments (arrows). DOX group. Masson's stain. H) Milder fibrosis areas in the MSC group (arrow). Masson's stain 
changes were more common in the endocardium, in all ventricular walls and in the interventricular septum. Zenker's necrosis was not observed in the control group, but was minimally present in the MSC group. In the DOX group, necrosis was observed in all cases. Multifocal distributed necrosis foci were observed mostly in areas close to hyaline degenerated muscle fibers. Necrosis was most commonly seen in all ventricular walls and deeper muscle fibers. In the statistical evaluation of necrosis, the DOX group was different from the other groups $(\mathrm{P}<0.05)$.

The statistical results from Masson's trichrome staining are given in Figure 1E. Fibrosis was detected mostly in the DOX group. The percentages of fibrosis in the control and MSC groups were lower (Fig. 1F-H). The DOX group was significantly different from the control and MSC groups $(\mathrm{P}<0.05)$, whereas the dif- ferences between the MSC and control groups were statistically insignificant $(\mathrm{P}>0.05)$.

Immunohistochemical results. Statistical results and findings from immunohistochemical staining are given in Table 1 and Figure 2. According to the results of the immunohistochemical staining of both anti-cTnT

Tab. 1. Immunohistochemical staining scores of the groups

\begin{tabular}{|l|c|c|c|}
\hline & $\begin{array}{c}\text { Control Group } \\
\text { (Mean } \pm \text { Std Error) }\end{array}$ & $\begin{array}{c}\text { DOX Group } \\
\text { (Mean } \pm \text { Std Error) }\end{array}$ & $\begin{array}{c}\text { MSC Group } \\
\text { (Mean } \pm \text { Std Error) }\end{array}$ \\
\hline CTnT & $61.350 \pm 1.375^{\mathrm{a}}$ & $6.891 \pm 1.001^{\mathrm{c}}$ & $24.771 \pm 1.342^{\mathrm{b}}$ \\
CTnl & $64.148 \pm 1.286^{\mathrm{a}}$ & $5.478 \pm 1.151^{\mathrm{c}}$ & $27.236 \pm 1.224^{\mathrm{b}}$ \\
C3 & $5.180 \pm 1.052^{\mathrm{b}}$ & $46.922 \pm 1.219^{\mathrm{a}}$ & $16.894 \pm 1.116^{\mathrm{b}}$ \\
BCL-XL & $11.021 \pm 1.145^{\mathrm{b}}$ & $6.890 \pm 1.046^{\mathrm{b}}$ & $21.976 \pm 1.171^{\mathrm{a}}$ \\
\hline
\end{tabular}

Explanations: $\mathrm{a}, \mathrm{b}, \mathrm{c}-$ means with different superscript letters differ significantly at $\mathrm{p} \leq 0.05$

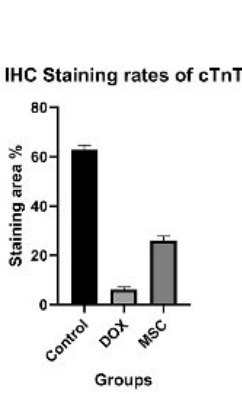

IHC Staining rates of $\mathrm{cTnl}$

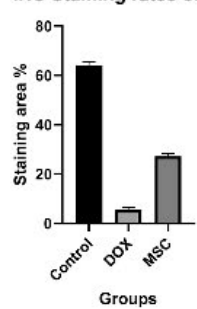

IHC Staining rates of BCL-XL

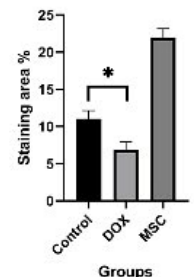

IHC Staining rates of Caspase-3

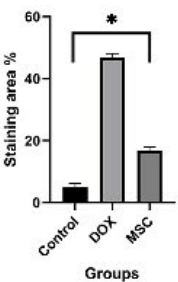

CONTROL

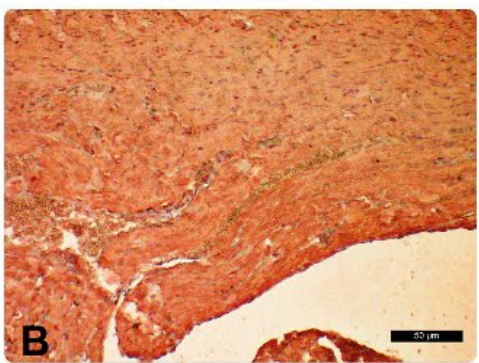

A

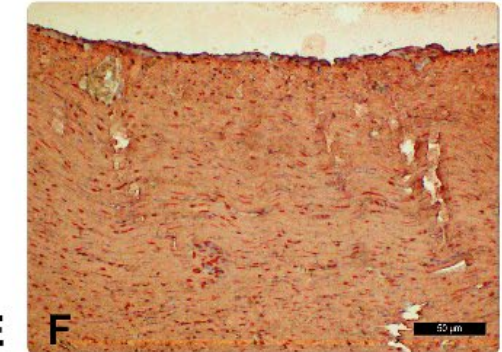

E
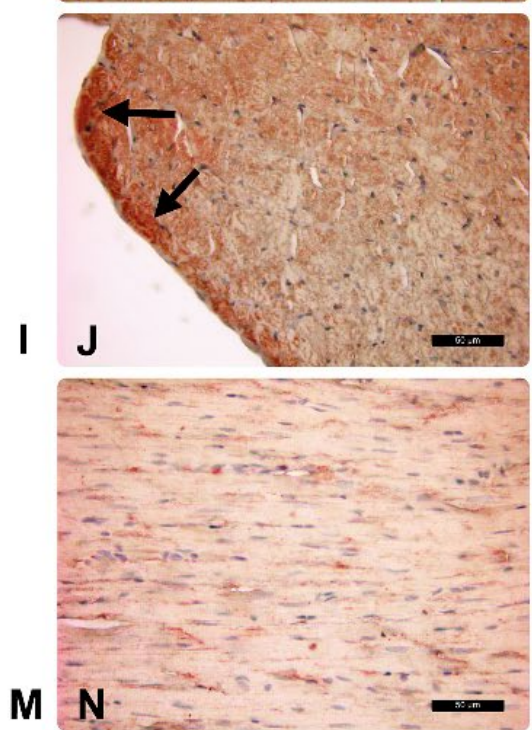

DOX
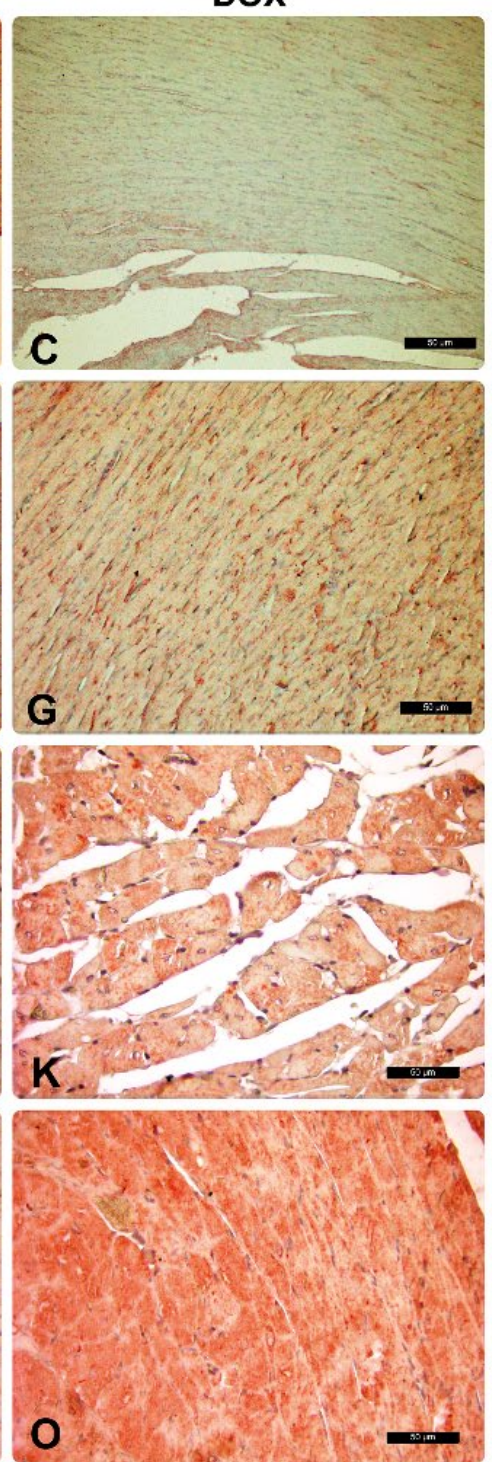

MSC
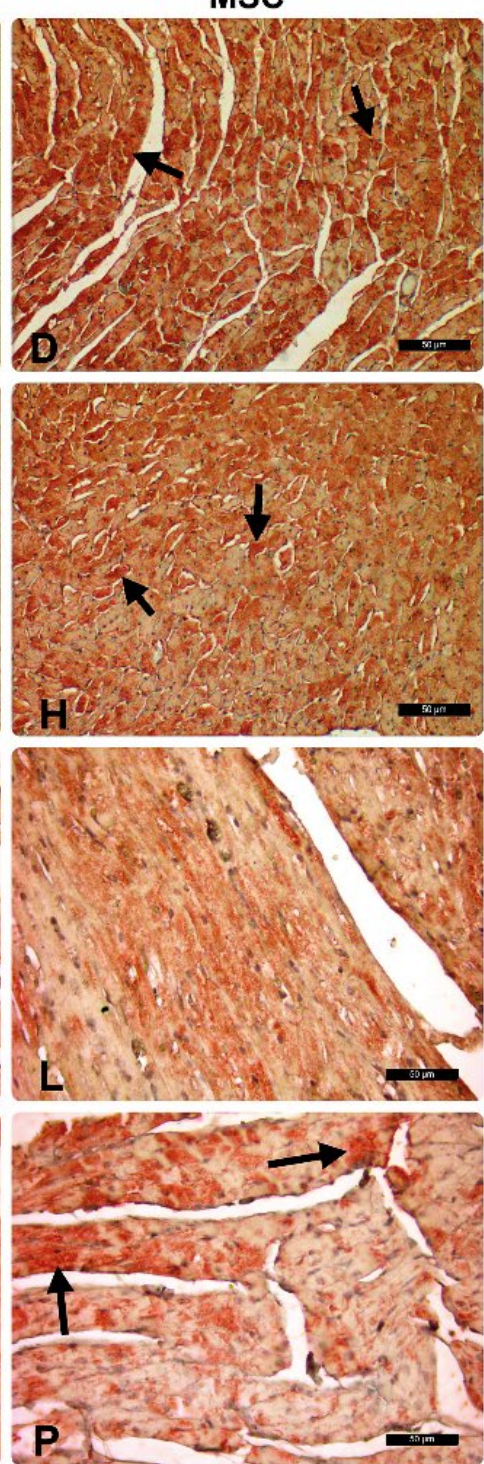

Fig. 2. Immunohistochemical appearance of the groups. A) Immunohistochemical staining rates for cTnT. B) Control Group. C) DOX Group. D) cTnT-positive immunostaining in muscle fibers (arrows). MSC Group. E) Immunohistochemical staining rates for cTnI. F) Control Group. G) DOX Group. H) cTnI immunopositive muscle fibers (arrows). MSC Group. I) Immunohistochemical staining rates for cTnT. BCL-XL. J) Positive staining in cardiac muscle filaments (arrows). Control Group. K) DOX Group. L) MSC Group. M) Immunohistochemical staining rates for Caspase-3. N) Control Group. O) DOX Group. P) Immunopositive reactions in muscle fibers (arrows). MSC Group. AEC. 
and anti-cTnI antibodies, the means were highest for the control group and lowest for the DOX group (Fig. 2A-H). The difference in staining between cTnT and cTnI was not statistically significant $(\mathrm{P}>0.05)$. Considering antibody staining individually, the mean value for the DOX group was different from those for the other groups $(\mathrm{P}<0.05)$. Immunonegative staining was not observed in the control group. In the DOX group, immunonegative staining was more common in muscle filaments in epicardial muscle fibers and middle parts of ventricular walls. Immunonegativity in the MSC group was diffuse and randomly distributed among muscle fibers. When histopathological sections were evaluated at the same time, negative immunoreactions were more intense in necrotic muscle fibers.

Caspase- 3 expression was highest in the DOX group and lowest in the control group. The mean values for the DOX group and the other groups were statistically significant $(\mathrm{P}<0.05)$ (Fig. $2 \mathrm{I}-\mathrm{L})$. On the other hand, while the statistical difference between BCL-XL expression in the control and DOX groups was not significant $(\mathrm{P}>0.05)$, this difference was significant in the MSC group [ $\mathrm{P}<0.05$ (Fig. 2M-P)].

Histopathological methods have been used in many studies to investigate the effect of Dox-IC in rats $(2,18)$. A previous study concluded that embryonic stem cell treatment prevents cytoplasmic vacuolization and myofibrillary losses by histopathology (17). In another study, it was found that bone marrow-derived MSCs reduce inflammatory cell infiltration in the heart (2). Recently, researchers using bone marrow-derived MSCs for therapeutic purposes found by histopathological examination that myofibril degeneration was decreased (21). In the present study, vacuole deposition, hyaline degeneration and necrosis were most frequent and severe in the DOX group, whereas the number and severity of lesions were lower in the MSC group. Statistically, the MSC and DOX groups were found to be different. It was therefore concluded that treatment with MSCs had a positive effect on histopathological findings.

Researchers have reported that fibrosis occurs in Dox-IC, but decreases after embryonic stem cell therapy (17). Data from another study suggests that intravenous administration of MSCs inhibits the development of fibrosis in Dox-IC (11). It has also been suggested that human-induced pluripotent stem cell treatment reduces fibrosis (22). In the present study, it was concluded that the treatment with FKD-MSCs prevented the development of fibrosis.

Cardiac troponins are proteins that regulate the contraction of myofibrils and are found in bundles of cardiac muscles. In the present study, the expressions of cTnT and cTnI proteins were shown to determine cardiac damage immunohistochemically. Immunoreactions in the MSC group were statistically different from those in the DOX group, but not at the same level as those in the control group. It is thought that troponins may be destroyed even during normal metabolism unless there is cardiac damage. In addition, recent studies have shown that stem cells do not convert directly into cardiomyocytes, and induce angiogenesis, immune regulation and production of paracrine factors (5). This confirms the fact that troponin expression in muscle filaments in the MSC group was statistically different from that in the control group. Immunohistochemical examinations showed that the expressions of cTnT and cTnI were similar, indicating that both biomarkers could be used to demonstrate myocardial damage.

In a previous study, there was no loss of troponin in non-necrotic myocardial tissue, and immunohistochemical analyses were more sensitive than histopathological examinations in cardiac damage (6). In the present study, immunohistochemical examinations revealed that the most intense troponin losses occurred in necrotic muscle fibers. Although, histopathologically, necrosis was not statistically different in the control and MSC groups, all groups were different in terms of troponins in immunohistochemical staining. This suggests that immunohistochemical analyses are more sensitive than histopathology.

Determination of apoptosis pathways has been reported to be one of the most effective methods of detecting Dox-IC (12). Following DOX administration, oxidative stress occurs due to increased levels of reactive oxygen species and free oxygen radicals, particularly intracellular calcium, in myofibers and endothelial cells in the heart, causing necrosis and apoptosis in cardiomyocytes (22). In the present study, the percentage of Caspase-3 staining was highest in the DOX group. However, it was thought that the stem cell therapy had a positive effect on apoptosis, since the expressions of Caspase- 3 in the control group and the MSC group were similar. Furthermore, the histopathological findings of Zenker's necrosis in the control and MSC groups were statistically similar, which supports this finding. BCL-XL acts as an anti-apoptotic factor and regulates many different cell death types, just as BCL-2 (10). According to previous studies on prevention of Dox-IC, administration of stem cells increases the expression of anti-apoptotic factors, such as BCL-2 (21). In the present study, BCL-XL expression was highest in the MSC group, and it was concluded that the stem cell therapy helped prevent apoptosis.

In previous studies, intravenous, subepicardial, intramyocardial and intracoronary methods have been used as routes of stem cell administration. However, only a few studies have attempted to quantify the impact of intraperitoneal administration of MSCs in Dox-IC cases (1). It was observed that the intraperitoneal administration of stem cells was positively reflected in histopathological findings (14). In the present study, the intraperitoneal route of administration was used, and, similar to the studies mentioned above, positive effects were obtained in histopathological examinations. However, comparison of the limited number of 
intraperitoneal administrations with other methods will provide more information about the reliability of this administrations route.

In conclusion, intraperitoneal administration of FKD-MSCs was found to reduce histopathological changes, such as vacuoles, degeneration, necrosis and fibrosis. It was also concluded from the immunohistochemical findings that it triggered apoptosis and antiapoptotic factors. However, further studies are required to determine other ways in which MSC administration prevents cardiac damage caused by Dox-IC.

\section{References}

1. Abushouk A. I., Abdo Salem A. M., Saad A., Afifi A. M., Afify A. Y., Afify H., Salem H. S. E., Ghanem E., Abdel-Daim M. M.: Mesenchymal stem cell therapy for doxorubicin-induced cardiomyopathy: Potential mechanisms, governing factors, and implications of the heart stem cell debate. Front Pharmacol. 2019, 10, 635, 1-12, doi: 10.3389/fphar.2019.00635.

2. Ammar H. I., Sequiera G. L., Nashed M. B., Ammar R. I., Gabr H. M., Elsayed H. E., Sareen N., Rub E. A., Zickri M. B., Dhingra S.: Comparison of adipose tissue- and bone marrow-derived mesenchymal stem cells for alleviating doxorubicin-induced cardiac dysfunction in diabetic rats. Stem. Cell Res. Ther. 2015, 6, 148, 1-14, doi: 10.1186/s13287-015-0142-x.

3. Arola O. J., Saraste A., Pulkki K., Kallajoki M., Parvinen M., Voipio-Pulkki L. M.: Acute doxorubicin cardiotoxicity involves cardiomyocyte apoptosis. Cancer Res. 2000, 60, 1789-1792.

4. Clements P., Brady S., York M., Berridge B., Mikaelian I., Nicklaus R., Gandhi M., Roman I., Stamp C., Davies D., McGill P., Williams T., Pettit S., Walker D., ILSI HESI Cardiac Troponins Working Group, Turton J.: Time course characterization of serum cardiac troponins, heart fatty acid-binding protein, and morphologic findings with isoproterenol-induced myocardial injury in the rat. Toxicol. Pathol. 2010, 38, 703-714, doi: 10.1177/0192623310374969.

5. Eschenhagen T., Bolli R., Braun T., Field L. J., Fleischmann B. K., Frisén J., Giacca M., Hare J. M., Houser S., Lee R. T., Marbán E., Martin J. F., Molkentin J. D., Murry C. E., Riley P. R., Ruiz-Lozano P., Sadek H. A., Sussman M. A., Hill J. A.: Cardiomyocyte regeneration: a consensus statement. Circulation 2017, 136, 680-686, doi: 10.1161/circulationaha.117.029343.

6. Fishbein M. C., Wang T., Matijasevic M., Hong L., Apple F. S.: Myocardial tissue troponins $\mathrm{t}$ and $\mathrm{i}$ : an immunohistochemical study in experimental models of myocardial ischemia. Cardiovasc. Pathol. 2003, 12, 65-71, doi: 10.1016 S1054-8807(02)00188-6.

7. Haydardedeoglu A. E., Boztok Ozgermen D. B., Yavuz O.: Mesenchymal stem cells reduce left ventricular mass in rats with doxorubicin-induced cardiomyopathy. Int. J. Morphol. 2018, 36, 48-53.

8. Ji M., Bai C., Li L., Fan Y., Ma C., Li X., Guan W.: Biological characterization of sheep kidney derived mesenchymal stem cells. Exp. Ther. Med. 2016, 12, 3963-3971, doi: 10.3892/etm.2016.3902.

9. Luna L. G. (ed.): Manual of histologic staining methods of the armed forces institute of pathology. $3^{\text {rd }}$ Edition, McGraw-Hill, New York 1968, p. 1-72.

10. Michels J., Kepp O., Senovilla L., Lissa D., Castedo M., Kroemer G. Galluzzi L.: Functions of BCL-XL at the interface between cell death and metabolism. Int. J. Cell. Biol. 2013, 705294, 1-10, doi: 10.1155/2013/705294.

11. Mohammadi Gorji S., Karimpor Malekshah A. A., Hashemi-Soteh M. B., Rafiei A., Parivar K., Aghdami N.: Effect of mesenchymal stem cells on Doxorubicin-induced fibrosis. Cell J. 2012, 14, 142-151, doi: 10.3389/ fimmu.2012.00212.

12. Mousa H. S. E., Abdel Aal S. M., Abbas N. A. T.: Umbilical cord blood-mesenchymal stem cells and carvedilol reduce doxorubicin- induced cardiotoxicity: Possible role of insulin-like growth factor-1. Biomed. Pharmacother. 2018, 105, 1192-1204, doi: 10.1016/j.biopha.2018.06.051.

13. Octavia Y., Tocchetti C. G., Gabrielson K. L., Janssens S., Crijns H. J., Moens A. L.: Doxorubicin-induced cardiomyopathy: From molecular mechanisms to therapeutic strategies. J. Mol. Cell. Cardiol. 2012, 52, 1213-1225, doi: 10.1016/j.yjmcc.2012.03.006.

14.Pinarli F. A., Turan N. N., Güçlü Plnarll F., Okur A., Sönmez D., Ulus T., Oğuz A., Karadeniz. C., Delibaşı T.: Resveratrol and adipose-derived mesenchymal stem cells are effective in the prevention and treatment of doxorubicin cardiotoxicity in rats. Pediatr. Hematol. Oncol. 2013, 30, 226-238, doi: 10.3109/08880018.2012.762962

15. Pittinger M. F., Martin B. J.: Mesenchymal stem cells and their potential as cardiac therapeutics. Circ. Res. 2004, 95, 9-20, doi: 10.1161/01. RES.0000135902.99383.6f.
16. Shi Y., Moon M., Dawood S., McManus B., Liu P. P.: Mechanisms and management of doxorubicin cardiotoxicity. Herz 2011, 36, 296-305, doi: 10.1007/ s00059-011-3470-3.

17. Singla D. K., Ahmed A., Singla R., Yan B.: Embryonic stem cells improve cardiac function in doxorubicin-induced cardiomyopathy mediated through multiple mechanisms. Cell Transplant. 2012, 21, 1919-1930, doi: 10.3727/096368911X627552.

18. Soliman A. N., Abd-Allah S. H., Hussein S., Alaa Eldeen M.: Factors enhancing the migration and the homing of mesenchymal stem cells in experimentally induced cardiotoxicity in rats. IUBMB Life. 2017, 69, 162-169, doi: 10.1002/ iub. 1600 .

19. Wallace K. B.: Doxorubicin-induced cardiac mitochondrionopathy. Pharmacol. Toxicol. 2003, 93, 105-115, doi: 10.1034/j.1600-0773.2003.930301.x.

20. Wang M., Yuan Q., Xie L.: Mesenchymal stem cell-based immunomodulation: properties and clinical application. Stem. Cells Int. 2018, 3057624, 1-12, doi: 10.1155/2018/3057624.

21. Zeng Y. L., Zheng H., Chen Q. R., Yuan X. H., Ren J. H., Luo X. F., Chen P., Lin Z. Y., Chen S. Z., Wu X. Q., Xiao M., Chen Y. Q., Chen Z. Z., Hu J. D., Yang T.: Bone marrow-derived mesenchymal stem cells overexpressing MiR-21 efficiently repair myocardial damage in rats. Oncotarget 2017, 8, 29161-29173, doi: 10.18632/oncotarget.16254.

22. Zhang Y., Liang X., Liao S., Wang W., Wang J., Li X., Ding Y., Liang Y., Gao F., Yang M., Fu Q., Xu A., Chai Y. H., He J., Tse H. F., Lian Q.: Potent paracrine effects of human induced pluripotent stem cell-derived mesenchymal stem cells attenuate doxorubicin-induced cardiomyopathy. Sci. Rep. 2015, 5, 11235, 1-17, doi: 10.1038/srep11235.

23. Zhang Y. W., Shi J., Li Y. J., Wei L.: Cardiomyocyte death in doxorubicininduced cardiotoxicity. Arch. Immunol. Ther. Exp. (Warsz). 2009, 57, 435-445, doi: 10.1007/s00005-009-0051-8.

Corresponding author: Orhan Yavuz, DVM, PhD, Assoc. Prof. Dr., Aksaray University, Faculty of Veterinary Medicine, Department of Pathology, 68100, Aksaray, Turkey; e-mail: vetorhanyavuz@gmail.com 